\title{
NÍVEL DE IMPLANTAÇÃO E ALCANCE DAS PRÁTICAS DA GESTÃO DO CONHECIMENTO EM BASE TECNOLÓGICA E FUNCIONAL DE ORGANIZAÇÃO EDUCACIONAL
}

\author{
LEVEL OF IMPLEMENTATION AND REACH OF KNOWLEDGE MANAGEMENT PRACTICES IN THE \\ TECHNOLOGICAL AND FUNCTIONAL BASE OF AN EDUCATIONAL ORGANIZATION
}

NIVEL DE IMPLEMENTACIÓN Y ALCANCE DE LAS PRÁCTICAS DE LA GESTIÓN DEL CONOCIMIENTO EN BASE TECNOLÓGICA Y FUNCIONAL DE UNA ORGANIZACIÓN EDUCATIVA

\author{
JULIANA DE CÁSSIA BENTO \\ Mestre \\ Centro Universitário de Maringá - Brasil \\ jcbento@hotmail.com \\ ARTHUR GUALBERTO BACELAR DA CRUZ URPIA \\ Doutor \\ Centro Universitário de Maringá - Brasil \\ arthurbacellar@yahoo.com.br \\ FLÁVIO BORTOLOZZI \\ Doutor \\ Centro Universitário de Maringá - Brasil \\ flavio.bortolozzi.53@gmail.com \\ ELY MITIE MASSUDA \\ Doutora \\ Centro Universitário de Maringá - Brasil \\ elymitie.m@gmail.com
}

Submetido em: 03/04/2017

Aprovado em: 22/06/2017

Doi: alcance.v24n2.p243-257

\section{RESUMO}

Este estudo analisou as práticas de gestão do conhecimento relacionadas à base tecnológica e funcional em instituição de ensino superior privada. Trata-se de uma pesquisa desenvolvida nos níveis exploratório e descritivo com abordagem quantitativa, cuja estratégia de pesquisa foi o estudo de caso único. $O$ instrumento de coleta dos dados foi o questionário desenvolvido pelo Instituto de Pesquisa Econômica Aplicada, que identifica o nível de implantação e o grau de alcance das práticas da gestão do conhecimento nas instituições. Foi aplicado em dezessete coordenadores de cursos de graduação na modalidade de educação a distância de uma Instituição de Ensino Superior (IES). Os resultados indicam que tanto o estágio de implantação como o nível de alcance das práticas relacionadas à base tecnológica e funcional na organização pesquisada são ainda incipientes. Conclui-se que a IES está em nível de maturidade baixo quanto à integração da Gestão do Conhecimento em suas atividades relacionadas à base tecnológica e funcional, o que prejudica a obtenção de vantagens competitivas.

Palavras-Chave: Organizações educacionais; diagnóstico de gestão do conhecimento; práticas de gestão do conhecimento. 


\section{ABSTRACT}

The study analyzes the knowledge management practices related to the technological and functional base in a private higher education institution. The research is exploratory and descriptive, with a quantitative approach, based on a single case study. The data collection instrument was a survey developed by the Instituto de Pesquisa Econômica Aplicada [Institute for Research in Applied Economics] was used, which identifies the level of implementation and reach of the knowledge management practices and actions in the institution. It was applied to seventeen undergraduate course coordinators in the distance education modality. The results indicate that both the implementation stage and the level of reach of the practices related to the technological and functional base in the organization studied are still incipient. We concluded that the IHE has a low level of maturity in terms of Knowledge Management integration in its activities related to the technological and functional base, which prevents it from obtaining competitive advantage.

Keywords: Educational organizations. Diagnostic knowledge management. Knowledge management practices.

\section{RESUMEN}

Este estudio analizó las prácticas de gestión del conocimiento relacionadas a la base tecnológica y funcional en una institución de enseñanza superior privada. Se trata de un estudio desarrollado en los niveles exploratorio y descriptivo con un abordaje cuantitativo, cuya estrategia de investigación fue el estudio de caso único. El instrumento de recolección de los datos fue el cuestionario desarrollado por el Instituto de Investigación Económica Aplicada, que identifica el nivel de implantación y el grado de alcance de las prácticas de la gestión del conocimiento en las instituciones. Fue aplicado a diecisiete coordinadores de cursos de grado en la modalidad de educación a distancia de una Institución de Educación Superior (IES). Los resultados indican que tanto el estadio de implementación como el nivel de alcance de las prácticas relacionadas a la base tecnológica y funcional en la organización estudiada son todavía incipientes. Se concluye que la IES está en un nivel de madurez bajo en lo que se refiere a la integración de la Gestión del Conocimiento en sus actividades relacionadas a la base tecnológica y funcional, lo que perjudica la obtención de ventajas competitivas.

Palabras clave: Organizaciones educacionales; Diagnóstico de gestión del conocimiento; Prácticas de gestión del conocimiento.

\section{INTRODUÇÃO}

Assim como as demais organizações, as IES são impulsionadas a inovar fazendo com que seu produto se torne mais atraente e diferenciado para manterem-se no mercado de forma competitiva e, principalmente, focadas nos resultados. A inovação é o caminho para alcançar vantagens competitivas, já que as organizações inovadoras tendem a ser mais flexíveis, têm maior capacidade de adaptação e de respostas às mudanças, conseguindo explorar as oportunidades existentes de maneira mais determinante que seus concorrentes (POSSOLLI, 2012).

Em função desta realidade, as organizações enfrentam hoje uma enorme concorrência e, para vencê-la, é necessário e fundamental ser competitiva e inovadora. Nas organizações educacionais não é diferente, pois em decorrência das rápidas mudanças ocasionadas pelo desenvolvimento das tecnologias da informação e da comunicação (TIC), tornou-se urgente se adequarem a este novo ambiente. No caso das IES, em decorrência disto e devido às demandas de novos conhecimentos e do uso das tecnologias nas universidades, há uma constante valorização do corpo técnico-administrativo envolvido na gestão universitária. Portanto, de uma forma geral, as organizações na era do conhecimento são instituições que aprendem e se diferenciam pelo que sabem e, principalmente, pela forma como conseguem usar o conhecimento.

Vale destacar que no Plano de Desenvolvimento Institucional (PDI) se definem a missão da IES e as estratégias para atingir suas metas e objetivos (BENTO, 2016). Abrangendo um período de cinco anos, deverá contemplar o cronograma e a metodologia de implementação dos objetivos, das metas e das ações do Plano da IES, observando a coerência e a articulação entre as diversas ações, a manutenção de padrões de qualidade e, quando pertinente, o orçamento. Deverá apresentar, ainda, um quadro-resumo contendo a relação dos principais indicadores de desempenho, que possibilite comparar, para cada um, a situação atual e futura (após a vigência 
do PDI). No PDI um dos indicadores obrigatórios e fundamentais é a sua infraestrutura. O PDI deve conter detalhadamente a relação de equipamentos, softwares e sistemas que permitem acesso dos docentes, técnicos e alunos aos equipamentos de informática ligados à sua rede de comunicação administrativa e científica, etc. Portanto, a base tecnológica disponível em uma IES não só é obrigatória como é fundamental para o gerenciamento: administrativo; econômico; financeiro e acadêmico. De um modo geral, esta dimensão fornece a base de informações de toda a IES e certamente colabora na tomada de decisão das ações. Logo, nesta dimensão, a GC é vital para o bom desenvolvimento das atividades universitárias ligadas ao funcionamento global da IES.

Nas IES privadas torna-se ainda mais importante o controle e a eficiência desta dimensão, pois ela responde pela criação, pelo armazenamento, pela distribuição e pelo compartilhamento das informações para 0 controle de todas as atividades da IES. Além disto, todo o controle das informações sobre os processos administrativos, fiscais, financeiros, acadêmicos e outros está sob a responsabilidade desta dimensão. Portanto, para a IES ser mais competitiva é fundamental que seus sistemas de informações que atuam nos processos, métodos e serviços sejam eficientes e eficazes.

Nas últimas décadas, as organizações passaram por período de grandes transformações e, mais recentemente, vivencia-se a transição da era da informação para a era do conhecimento (SCHREIBER, 2015). Para se adaptar e sobreviver a essa nova realidade, os indicadores econômicos de uma organização se tornaram vitais no processo decisório. A gestão do conhecimento (GC) tem como objetivo melhorar os processos das organizações e servir de base para a tomada de decisões. Neste cenário, conhecer o termo GC é importante. São muitos os autores que atuam nesse campo, cujos conceitos de alguns deles são apresentados a seguir: um processo, pelo qual as organizações buscam novas formas de criar e expandir o conhecimento (NONAKA; TAKEUCHI, 1997); uma metodologia de gerenciamento que se preocupa com as estratégias para alcançar a inteligência competitiva (CHAPARRO, 1998); um conjunto de ações que procura identificar, capturar, gerenciar e compartilhar as informações da organização (DAVENPORT; PRUSAK, 1998); uma nova área na união entre a tecnologia de informação e administração, um campo novo entre a estratégia, a cultura e os sistemas de informação de uma organização (TEIXEIRA FILHO, 2000); a criação, a disseminação e a utilização do conhecimento a partir de um conjunto de processos organizacionais (ANGELONI, 2002); o núcleo de organização de ativos intangíveis o qual interfere positivamente o desempenho de uma empresa (SULLIVAN, 2005).

Diversos estudos vêm indicando a aplicação da GC em organizações brasileiras: o diagnóstico da GC nas organizações públicas (FRESNEDA et al., 2009); a GC e a visão cognitiva dos repositórios institucionais (FACHIN et al., 2009); a inovação organizacional e a inovação de modelo de negócios (FIGUEIRA et al. 2011); 0 uso de modelo de GC para produzir resultados em benefício do cidadão (BATISTA, 2012); a GC em empresa internacional de energia (BRITO et al. ,2013); as ferramentas de avaliação de GC em estudos bibliométricos (FREIRE et al., 2013); a análise do nível de maturidade da GC na criminalística federal (MENDES, 2013); as metodologias para identificação de modelos de maturidade em GC para a aplicação empírica (NATALE et al., 2014); a gestão do conhecimento nas escolas da Amusep (URPIA et al., 2016); a gestão do conhecimento e a inovação em governo (AGUNE, 2014); a análise do nível de implantação e o alcance das práticas da gestão do conhecimento em uma instituição privada de ensino superior (BENTO et al., 2016).

Este estudo tem como objetivo analisar as práticas de GC relacionadas à base tecnológica e funcional em uma instituição de ensino superior privada que atua na modalidade de ensino a distância no norte do Paraná. Este trabalho justifica-se, tendo-se em vista que, no Brasil, a expansão do sistema universitário nos últimos anos vem trazendo uma série de novas exigências para as IES. Dentre elas, Bolson (2012) cita a necessidade de os dirigentes universitários atuarem em vários setores da Instituição, com competência para superarem os desafios impostos por um ambiente caracterizado por sua complexidade e com objetivos voltados para a criação e a difusão do conhecimento.

Além desta introdução, o presente artigo possui mais quatro seções: referencial teórico, metodologia, resultados e discussão e considerações finais.

\section{REFERENCIAL TEÓRICO}

\subsection{A Gestão do Conhecimento nas Organizações}

A acumulação do capital após a segunda revolução industrial se baseava no fordismo, que tinha como principal característica o consumo em massa. Para tal, o fordismo apresentou como principais inovações a linha de montagem automatizada com as esteiras rolantes e a valorização do salário dos trabalhadores mais produtivos,

Revista Alcance - Eletrônica - vol. 24 - n. 2 - abr./jun. 2017 
com o "Five dollars day". A partir de 1970, com o declínio (a própria rigidez do modo de produção industrial fordista é vista como o principal fator do seu declínio) do fordismo, fez-se necessário o desenvolvimento de um novo modo de acumulação do capital.

A terceira fase da revolução industrial, ou revolução técnico-científica, com as inovações de produtos e processos, provocou uma forte alteração no capitalismo. A revolução técnico-científica, que alterou as bases produtivas da economia a partir da década de 1970, tem seus fundamentos relacionados, de um lado, pela emergência das tecnologias da microeletrônica e da transmissão de informações e sobre a automatização e a robotização dos processos produtivos, de outro. A base produtiva da economia passou, então, a ser pautada por dois fatores: conhecimento e inovações. Esta mudança na base produtiva da economia provocou uma forte alteração na forma de acumulação do capital.

Com isto, as organizações passaram a enfrentar o desafio diuturnamente de manter-se competitiva, principalmente num cenário de constantes mudanças e instabilidade econômica. Por isso, possuir a habilidade de gerenciar os processos de criação do conhecimento, de forma que a organização possa deter conhecimentos que não estejam disponíveis para seus concorrentes, torna-se relevante para criar e sustentar vantagens competitivas. Porter (1999), em seu estudo sobre competição e estratégias, apresenta as três estratégias competitivas genéricas, são elas: i) liderança no custo, que é uma estratégia implementada por instituições que objetivam a ampliação da sua participação no mercado (market share); ii) diferenciação, que é uma estratégia que permite que as empresas ofertem produtos e serviços variados e com mais qualidade, atendendo assim a uma maior demanda do mercado; iii) enfoque, que visa a um nicho específico do mercado, contribuindo para explorar necessidades específicas dos seus consumidores. Para a implementação dessas estratégias, são importantes investimentos em pesquisa e desenvolvimento por parte das instituições. Segundo Porter (1999), uma vez introduzidas essas estratégias genéricas, as empresas conseguirão obter vantagens competitivas e, com isto, terão mais facilidade para lidar com as forças competitivas do mercado: poder de barganha dos fornecedores, ameaça de produtos substitutos, ameaça de novos entrantes, poder de barganha dos clientes e rivalidade entre concorrentes. Schreiber (2015) ressalta papel estratégico da inovação e da GC nas novas tendências para o diferencial competitivo, suplantando-se o estágio das vantagens de produção baseada na escala e no escopo.

Faz-se importante definir competitividade para identificar seus fatores preponderantes. A competitividade apresenta-se figurada na produtividade das empresas e é ligada à capacidade dos governos, ao comportamento da sociedade e aos recursos naturais, permitindo conquistar e assegurar posições no mercado. Para se manter competitiva, considerando a escassez de recursos e a nova economia, faz-se necessário a utilização de novas tecnologias, o que possibilita às empresas ter capacidade de compensar os fatores escassos por meio de novos produtos e processos (COUTINHO; FERRAZ, 1995; STEFANO et al., 2014).

Segundo Alee (1997), a organização precisa ter a habilidade necessária para localizar as fontes de conhecimento especializado para manter-se competitiva, continuar a gerar lucros e ainda utilizar o conhecimento existente na organização para resolução de problemas ou tomada de decisão. Nesse sentido, conforme Davenport, De Long e Beers (1998, p. 43), "conhecimento é informação combinada com experiência, contexto, interpretação e reflexão". O conhecimento pode assumir representações tangíveis (que caracteriza 0 conhecimento explícito) e intangíveis (que caracteriza o conhecimento tácito) (RODRIGUES; GRAEM, 2013).

Segundo Choo (2003), existem três áreas onde a criação e o uso da informação se apresentam como ação estratégica para a obtenção de competitividade pelas organizações. "Primeiro, a organização usa a informação para dar sentido às mudanças no ambiente externo" (CHOO, 2003, p. 27). Desta forma, a empresa identifica e dá sentido às mudanças do ambiente externo por meio da informação para tomar decisões e traçar ações, com o objetivo de tornar-se adaptável ao cenário dinâmico, de constantes mudanças.

A segunda área estudada e elencada por Choo (2003) é a de criar, organizar e processar a informação, a fim de gerar conhecimentos, garantindo o aprendizado organizacional. Para Choo (2003, p.28), "novos conhecimentos permitem à organização desenvolver novas capacidades, criar novos produtos e serviços, aperfeiçoar os já existentes e melhorar os processos organizacionais". Isso se torna possível quando a organização entende a importância da sinergia existente entre o conhecimento tácito e explícito e quando são elaboradas práticas para conversão desse intrínseco para oficial, dando origem a novos conhecimentos.

A terceira área de utilização da informação como recurso estratégico para a organização é na tomada de decisões, quando ela é baseada na criação de significados anteriormente explicados e também da construção dos conhecimentos para a ação (CHOO, 2003). 
Nonaka e Takeuchi (1997), ao analisarem como as organizações se mantêm competitivas gerando inovação, sugerem que as empresas, além de processar o conhecimento, também o criam. Schumpeter (1997, p. 9) conceitua inovação e coloca o empresário como figura importante no processo, considerando o empresário inovador como "agente econômico que traz novos produtos para o mercado por meio de combinações mais eficientes dos fatores de produção, ou pela aplicação prática de alguma invenção ou inovação tecnológica". Nesse sentido, o autor considera a liderança da organização a chave principal de mudança e possível inovação.

Nonaka e Takeuchi (1997) atribuem à organização as bases para o entendimento dos processos de criação do conhecimento organizacional. 0 conhecimento explícito, que pode ser codificado e formalizado facilmente na organização, apresenta-se como uma parte do conhecimento individual, pois a maior parte está contida no conhecimento tácito, caracterizado pela parte cognitiva do indivíduo, experiências vividas e modelos mentais. Diante disso, os autores pressupõem que o conhecimento é criado e difundido na organização por meio da interação social entre o conhecimento tácito e explícito, num processo de conversão do conhecimento.

Assim, a espiral de interações entre o conhecimento tácito e explícito constitui um processo de criação de conhecimento principal. Isso possibilita que o conhecimento tácito seja explicitado à medida que representa compreensões intuitivas na forma de metáforas, analogias, conceitos, hipóteses e modelos (NONAKA; TAKEUCHI, 1997). Uma vez que o conhecimento tácito alcançou o estado de explicitação, pode ser compartilhado e tornar-se parte de um repertório de conhecimento mais amplo que os colaboradores utilizam para transformar 0 projeto e o fornecimento de produtos e serviços (OLIVEIRA; CARVALHO, 2015).

A criação do conhecimento é um processo intencional e tem o propósito de fomentar ativos organizacionais, sendo o conhecimento tácito ferramenta fundamental para a inovação e a competitividade e seu compartilhamento é fonte de crescimento organizacional contínuo (NONAKA; TAKEUCHI, 2008).

Diante da importância do conhecimento e da GC organizacional para a competitividade das organizações, diversas ferramentas da GC foram desenvolvidas e incorporadas pelas organizações.

\subsection{Ferramentas da Gestão do Conhecimento}

As ferramentas de GC agrupadas na categoria de base tecnológica e funcional da organização apresentam como objetivo dar suporte à GC organizacional, incluindo a automação da gestão da informação, dos aplicativos e das ferramentas de Tecnologia da Informação (TI) para captura, difusão e colaboração do conhecimento. Algumas ações e práticas se destacam nesta categoria.

A primeira prática a ser explanada é gestão eletrônica de documentos (GED). Esta ferramenta caracteriza-se pela utilização da TI, por meio de aplicativos de controle de emissão, edição e acompanhamento da tramitação, distribuição, arquivamento e descarte de documentos. Para Koch (1997, p. 23), "o GED visa gerenciar 0 ciclo de vida das informações desde sua criação até 0 seu arquivamento. As informações podem originalmente estar armazenadas em mídias analógicas ou digitais em todas as fases de sua vida". Essa prática da GC assegura que o conhecimento produzido na contemporaneidade seja transmitido para as gerações futuras, sempre que acessado.

Ferramentas de colaboração, como portais, intranets e extranets, são definidas por Batista (2006) como práticas que capturam e difundem conhecimento e experiência entre trabalhadores e departamentos por meio de sistemas informatizados e portais. Tais ferramentas baseiam-se em portal de informação que acelera o processo de aprendizagem e facilita a transferência eficaz entre as formas de conhecimento tácito e explícito, pois contêm informações estruturadas, redes e comunidades, fóruns de discussão do conhecimento e espaços de trabalho colaborativos, visando incentivar e transferir de forma espontânea a troca de conhecimento tácito (YOUNG, 2010). Entende-se por portal um espaço virtual que integra sistemas corporativos com segurança e privacidade de dados organizacionais, caracterizando-se em um ambiente de trabalho e repositório de conhecimento para a organização e seus colaboradores, proporcionando acesso a todas as informações e aplicações relevantes, assim como uma plataforma para comunidades de prática, redes de conhecimento e melhores práticas (BATISTA, 2006).

A prática sistema de workflow caracteriza-se pela automação de processos de controle interno ou trâmite de documentos, normalmente implantado para simplificar e agilizar o processo. 0 sistema auxilia e dá suporte ao controle de qualidade, otimizando os fluxos de trabalho. Segundo Batista (2006, p. 29), o workflow "(..) é utilizado para controle de documentos e revisões, requisições de pagamentos, estatísticas de desempenho de funcionários, etc.". Caracteriza-se como uma ferramenta da TI para apoio à GC. 
Data warehouse ou armazém de dados se apresenta como uma ferramenta da TI de apoio à GC. Para Batista (2006, p.59), trata-se do "(...) rastreamento de dados com arquitetura hierarquizada disposta em bases relacionais, a qual permite versatilidade na manipulação de grandes massas de dados". A prática possibilita gerenciar as informações, independente do volume de dados, visando à facilidade de acesso e ao manuseio das mesmas. Kimball (1998) define a prática data warehouse como uma cópia das transações e dos dados da organização disponibilizada somente para leitura, estruturada de maneira que permita análise e consulta. Essa prática implantada permite ao gestor acesso às informações de maneira rápida e intuitiva, otimizando o processo de tomada de decisão, cujo julgamento humano é fundamental, porém a limitação humana para processar as informações dificulta o processo.

A prática data mining, ou mineradores de dados, apresenta-se como ferramenta da TI para suporte à GC. Segundo Batista (2006, p. 59), os mineradores de dados caracterizam-se por "instrumentos com alta capacidade de associação de termos, o que lhes permite 'garimpar' assuntos ou temas específicos". Esta prática é aplicada, juntamente com a data wirehouse, com o intuito de facilitar a busca de informações, pois é capaz de revelar de maneira automática o conhecimento que se encontra implícito em grandes quantidades de dados e informações armazenadas em banco de dados da organização (KING, 2003). Para Elmasri e Navathe (2002), a ferramenta de data mining compreende os seguintes propósitos: de previsão, pois mostra como certos atributos dos dados se comportarão no futuro; identificação de dados, para localização de um evento ou atividade; classificação dos dados, de modo que diferentes categorias possam ser identificadas; e otimização do uso de recursos limitados.

Gestão de conteúdo apresenta-se, segundo Batista (2006), como um processo de seleção, captura, classificação, indexação, registro e depuração de informações dentro da organização, por meio de websites, wikis, blogs, portais corporativos. Ou seja, é uma ferramenta que busca soluções para simplificar os processos de criação, utilização, disseminação e armazenamentos de conteúdos. Para Boiko (2001), os sistemas de gestão de conteúdo definem-se como um ambiente de trabalho colaborativo que fornece suporte para realização das tarefas desempenhadas pelas pessoas, baseado em um conjunto de processos que deve ser estruturado para a produção de publicações digitais.

Para atingir esse objetivo, o sistema de gestão de conteúdos deve apresentar uma interface intuitiva e de fácil navegação para que qualquer colaborador possa gerar seus conteúdos e publicá-los de qualquer lugar, a qualquer momento (PARREIRAS, 2005). É conveniente ressaltar que o sucesso dessa prática depende não somente da TI, mas primordialmente das pessoas envolvidas, dependendo delas a retroalimentação do conteúdo gerado no sistema. Além disso, aspectos culturais e comportamentais da organização devem ser considerados, tendo em vista a disponibilização do conhecimento produzido para os parceiros, incluindo aspectos de gestão de documentos e de processos de trabalho (BOIKO, 2001).

A ferramenta costumer relationship management (CRM) caracteriza-se pela gestão de relacionamento com o cliente que coleta os dados detalhados oriundos da organização em transação com os clientes e os transforma em informações e conhecimento para utilização no planejamento dos gestores. Cada cliente tem seu perfil, que demanda necessidades únicas para empresa, desta forma, com a utilização de uma ferramenta como - CRM, a organização pode direcionar seus esforços para captação dos clientes que identifica ser de maior valor e ainda personalizar seu comportamento baseando-se nas necessidades individuais buscados pelos clientes (FINGER; CASTRO, 2004). Pode-se inferir, portanto, que rastrear constantemente o comportamento e as necessidades dos clientes é objetivo da ferramenta de CRM.

A prática balanced scorecard (BSC), original da área de contabilidade por utilizar indicadores financeiros em sua essência, na contemporaneidade, pode ser entendida como um sistema que considera inclusive indicadores não financeiros oriundos do planejamento da organização. O diferencial do BSC é a capacidade de comunicar a visão e a estratégia da empresa por meio de indicadores de desempenho de estratégias e metas. Essa comunicação baseia-se no gerenciamento de metas estabelecidas, alcançadas ou não, dando ao gestor a possibilidade de realocar recursos para alcançar os objetivos estratégicos (KAPLAN; NORTON, 1997). O objetivo do BSC, segundo Kaplan e Norton (1997), resume-se em tornar a estratégia, a missão e os valores da organização acessíveis e de fácil entendimento a todos os colabores e gestores, para canalizar os esforços a fim de evitar a dispersão de ações e fazer com que todos saibam o que fazer e de que forma suas ações impactam no desempenho organizacional.

0 decision support system (DSS) caracteriza-se como o sistema de suporte à tomada de decisão, baseado na $\mathrm{TI}$, que apoia a construção, a análise do modelo de raciocínio sobre a estrutura de um problema 
organizacional, ou simplesmente a escolha e a otimização de variáveis para tomada de decisão (DRUZDZEL; FLYNN, 2002). Esta ferramenta apresenta-se comumente utilizada pelos gestores de alto nível da organização.

A prática de Enterprise Resource Planning (ERP), ou sistemas integrados de gestão, apresenta-se como solução capaz de integrar as informações da empresa por meio de uma única base de dados. Para Buckhout, Frei e Nemec (1999), um ERP caracteriza-se pelo planejamento de recursos empresariais que integra as diferentes funções da organização com o objetivo de elaborar processos mais eficientes e melhorar processos de negociação usando a TI. A fim de obter sucesso na implantação da ferramenta, o entendimento de que se trata de um projeto empresarial e não somente tecnológico faz-se necessário. Todos os envolvidos no projeto e o seu engajamento são determinantes no processo de implantação e desenvolvimento da ferramenta, pois necessitam manusear 0 sistema (BENTO et al., 2016).

A prática Key Performance Indicators (KPI) (ou Indicador-chave de performance) é uma forma de medir se uma ação ou um conjunto de iniciativas estão atendendo aos objetivos propostos efetivamente. Os KPI se configuram como aplicativos tecnológicos capazes, que auxiliam o gestor na tomada de decisão. Devido ao fluxo constante e vasto de informações ao qual o gestor é submetido na organização, o desafio apresenta-se em quais indicadores escolher para acompanhar e medir (NADER; TOMI; PASSOS, 2012). Segundo os autores, esses indicadores podem ser mapeados no decorrer das atividades, inclusive ao longo do processo produtivo e medidos em situações e tempos diferentes, permitindo que a análise dos benefícios das atividades possa ser quantificada de maneira a subsidiar a tomada de decisão quanto a investimentos necessários. No ambiente pedagógico em que as organizações de ensino superior estão inseridas, os indicadores comumente utilizados, além dos mecanismos de avaliação externos, apresentam-se por meio das Comissões Próprias de Avaliação e Exame Nacional de Desempenho dos Estudantes. Essas avaliações são estabelecidas pelo Sistema Nacional de Avaliação da Educação Superior (URPIA et al., 2016).

As técnicas ou práticas aqui explicitadas podem contribuir de alguma forma para GC e para elevar o nível de aprendizagem nas organizações, cabendo-lhes optarem por aquelas que estão de acordo com suas necessidades, contribuindo para que a GC seja um processo dinâmico e contínuo.

\section{METODOLOGIA}

A presente pesquisa classifica-se como exploratória, de abordagem qualitativa e quantitativa. Como procedimento para coleta de dados, adotou-se um estudo de caso de instituição de ensino superior. Para preservar o sigilo das informações coletadas, a instituição foi denominada de 'IES X'. Os dezessete coordenadores de cursos de graduação na modalidade EAD, que são os gestores dos cursos de graduação, participaram da pesquisa.

$O$ instrumento de coleta de dados adotado foi um questionário desenvolvido pelo Instituto de Pesquisa Econômica Aplicada (IPEA)1. A escolha deste questionário justifica-se pela sua validação em estudos realizados em diversas áreas: Batista (2006, 2012); Batista et al., (2005); Batista et al., (2007); Batista et al., (2014). 0 questionário refere-se a uma lista com 27 práticas ou ações da GC que permite a identificação do grau de alcance e do estágio de implantação nas instituições. A aplicação do instrumento de pesquisa ocorreu no mês de novembro de 2015.

Faz-se importante destacar que, antes de serem submetidos ao questionário, todos os coordenadores de cursos foram individualmente capacitados, por meio de orientações a respeito de cada uma das 27 práticas e ações. Também receberam uma lista contendo as definições de cada uma das práticas e ações para consultar durante as suas respostas.

Seguindo a classificação utilizada por Batista $(2006,2012)$ e Batista et al. $(2005,2007,2014)$, as práticas e as ações contidas no questionário podem ser agrupadas em três categorias que estão representadas no Quadro 1:

\footnotetext{
1 O questionário utilizado na pesquisa está disponivel no site do IPEA (http://www.ipea.gov.br/ portal/images/stories/PDFs/TDs/td_1316.pdf).
} 
Quadro 1: Categorias das práticas e das ações contidas no instrumento de coleta de dados

\begin{tabular}{|l|l|}
\hline $\begin{array}{l}\text { Práticas e ações relacionadas aos aspectos de gestão } \\
\text { de recursos humanos. }\end{array}$ & $\begin{array}{l}\text { Tais práticas facilitam a transferência, a disseminação } \\
\text { e o compartilhamento de informações e de } \\
\text { conhecimento. }\end{array}$ \\
\hline $\begin{array}{l}\text { Práticas e ações ligadas à estruturação dos processos } \\
\text { organizacionais. }\end{array}$ & $\begin{array}{l}\text { Estas práticas e ações funcionam como facilitadores } \\
\text { de geração, retenção, organização e disseminação do } \\
\text { conhecimento organizacional. }\end{array}$ \\
\hline $\begin{array}{l}\text { Práticas e aç̃̃es cujo foco central é a base tecnológica } \\
\text { e funcional }\end{array}$ & $\begin{array}{l}\text { Estas práticas servem de suporte à GC } \\
\text { organizacional, incluindo a automação da gestão da } \\
\text { informação, dos aplicativos e das ferramentas de Tl } \\
\text { para captura, difusão e colaboração. }\end{array}$ \\
\hline
\end{tabular}

Fonte: Elaboração própria com base em Batista $(2006,2012)$ e Batista et al. $(2005,2007,2014)$.

Para alcançar o objetivo geral desse trabalho, apenas a última categoria foi analisada. Para isso, verificouse que as seguintes práticas contidas no questionário se relacionavam com essa última categoria. São elas: Portais/intranets/extranets; Sistemas de workflow; Gestão de conteúdo; GED; Data warehouse; DSS; BSC; Data mining; CRM; KPI e ERP.

Para a análise do estágio de implantação e do nível de alcance das ferramentas de GC, as seguintes escalas foram utilizadas:

a) O estágio de implantação das práticas e ações.

$0 \quad$ Não existem planos para implementação da prática.

1 Existem ações planejadas para a implementação da prática no futuro.

2 A prática está em processo de implementação.

3 A prática já está implantada.

4 A prática já está implantada e apresenta resultados importantes e relevantes para a organização.

b) O nível de alcance dentro da organização das práticas e das ações.

1 Poucas iniciativas isoladas dentro da organização.

2 Alguns departamentos usam a prática.

3 Muitos departamentos usam a prática.

4 Amplamente disseminada na organização.

Foi usado, nos dois casos, N/R para os não respondentes.

Uma vez aplicado o questionário, os dados foram organizados por meio do Programa Microsoft Excel (2010) e uma abordagem quantitativa foi aplicada. Os resultados foram apurados através de técnicas estatísticas: análise de distribuição de frequência absoluta e relativa dos dados. Para analisar os resultados obtidos, utilizouse a seguinte escala (Tabela 1): 
Tabela 1: Escala para a análise dos resultados

\begin{tabular}{l|l}
\hline Poucas & Menos de $25 \%$ dos departamentos têm iniciativas \\
Alguns & Mais de $25 \%$ e menos de $50 \%$ dos departamentos usam a prática \\
Muitos & Mais de $50 \%$ e menos de $75 \%$ usam a prática \\
Amplamente & Mas de $75 \%$ dos departamentos usam a prática \\
\hline
\end{tabular}

Fonte: Batista (2006). Elaborada pelos autores.

\section{RESULTADOS E DISCUSSÃO}

Para a análise e discussão dos resultados, dividiu-se este capítulo em duas seções: a primeira consiste em análise do estágio de implantação das práticas relacionadas à base tecnológica e funcional; a segunda identifica o nível de alcance nas organizações das práticas relacionadas à base tecnológica e funcional.

\subsection{Análise do Estágio de Implantação das Práticas.}

De acordo com a Tabela 2, ressalta-se que na IES X, para 64,7\% dos coordenadores, não existem planos para implementação, existindo apenas ações planejadas para a implementação da prática de GED no futuro. Entretanto existe um gargalo proveniente do arquivamento de provas e de documentos importantes no processo pedagógico que necessita de um tempo considerável para poder ser eliminado, uma vez que o espaço para a conservação do documento físico precisa ser relativamente grande devido ao volume e à quantidade de papel. $\mathrm{A}$ aplicação efetiva da GED, neste contexto, proporcionaria a IES X um ganho de espaço, tempo e agilidade nos processos de identificação e possível consulta desses documentos.

Tabela 2: Estágio de implantação das práticas e das ações relacionadas à base tecnológica e funcional.

\begin{tabular}{|l|c|c|c|c|c|c|}
\hline \multirow{2}{*}{ AÇÃO OU PRÁTICA } & \multicolumn{7}{|c|}{ ESTÁGIO DE IMPLANTAÇ̃̃O } \\
\cline { 2 - 7 } & $\mathbf{0}$ & $\mathbf{1}$ & $\mathbf{2}$ & $\mathbf{3}$ & $\mathbf{4}$ & N/R \\
\hline Ferramentas de colaboração como portais, intranets e extranets & 29,4 & 11,8 & 11,8 & 5,9 & 35,3 & 5,9 \\
\hline Sistemas de workflow & 23,5 & 5,9 & 23,5 & 11,8 & 29,4 & 5,9 \\
\hline Gestão de conteúdo & 41,2 & 23,5 & 23,5 & 5,9 & 0,0 & 5,9 \\
\hline Gestão Eletrônica de Documentos (GED) & 35,3 & 29,4 & 17,6 & 5,9 & 5,9 & 5,9 \\
\hline Data Warehouse (ferramenta da TI para apoio à GC) & 23,5 & 29,4 & 17,6 & 0,0 & 17,6 & 11,8 \\
\hline Data Mining (ferramenta da TI para apoio à GC) & 35,3 & 23,5 & 23,5 & 0,0 & 11,8 & 5,9 \\
\hline Costumer Relationship Management (CRM) & 5,9 & 17,6 & 35,3 & 5,9 & 11,8 & 23,5 \\
\hline Balanced Scorecard (BSC) & 29,4 & 23,5 & 0,0 & 5,9 & 5,9 & 35,3 \\
\hline Decision Suport System (DSS) & 29,4 & 17,6 & 5,9 & 11,8 & 0,0 & 35,3 \\
\hline Enterprise Resource Planning (ERP) & 23,5 & 11,8 & 11,8 & 5,9 & 11,8 & 35,3 \\
\hline KeyPerformance Indicator (KPI) & 23,5 & 29,4 & 0,0 & 5,9 & 5,9 & 35,3 \\
\hline Médias & 27,3 & 20,3 & 15,5 & 5,9 & 12,3 & 18,7 \\
\hline
\end{tabular}

Fonte: Elaborada pelos autores a partir da aplicação do questionário na IES X.

Verifica-se que, para $52,9 \%$ dos respondentes, não existem planos para implementação, mas há planejamento para implantação da prática data warehouse no futuro. 0 estágio de implantação da prática entre os coordenadores de curso ainda é pequeno, mas se pode inferir que a IES X compactua com a ideia de Kimball (1998) e de Batista (2006) no que tange à implantação da prática, pois permite ao gestor acesso de maneira rápida e intuitiva aos dados da organização, contribuindo para a tomada de decisão. Ainda que os departamentos sejam essencialmente pedagógicos, cada vez mais é exigido dos coordenadores de curso o direcionamento estratégico de planejamento de gestão dos cursos.

Nota-se que, para $58,8 \%$ dos respondentes, não existem planos para implementação, mas há planejamento para implantação da prática de data mining no futuro. Portanto se percebe que entre os 
coordenadores de curso, a prática ainda não é utilizada. Entretanto, para $47 \%$ dos coordenadores, a prática está sendo planejada ou está em processo de implantação. Isso significa que se torna crescente a preocupação da IES $\mathrm{X}$ em melhor gerenciar as informações, tornando-as conhecimento, facilitando a tomada de decisão por parte dos gestores, conforme defendido por King (2003), Elmasri e Navathe (2002) e Batista (2006).

A prática CRM, para $52,9 \%$ dos coordenadores, está sendo planejada ou está em processo de implantação. Destaca-se que o CRM é um sistema que permite o contínuo e efetivo contato com os clientes de uma organização, facilitando e agilizando o tratamento das demandas apresentadas por eles e a geração de informação para a instituição. Essa é uma prática importante do ponto de vista estratégico para a competitividade da IES X por facilitar a sua relação com os seus clientes, que são seus alunos. Entretanto, salienta-se que o CRM é um sistema computacional de alto custo financeiro.

O estágio de implantação das práticas e das ações de BSC, DSS e KPI, para quase metade dos coordenadores, é baixo, pois eles afirmaram que não existem planos para implementação dessas práticas ou existem apenas ações planejadas para a implementação futura delas.

No que diz respeito à prática BSC, infere-se que é uma prática restrita a alguns departamentos na IES X. No setor pedagógico, a utilização do BSC contribui para o alinhamento das estratégias da organização e das metas estabelecidas pelo departamento, tornando-os de fácil acesso a todos os colaboradores, conforme explanado por Kaplan e Norton (1997).

Verifica-se que existem muitas dificuldades no nível de implantação das práticas de gestão de conteúdo, GED e Data Mining. Tais dificuldades podem ser explicadas pelo fato da IES $X$ ter se empenhado na implementação das bases de TI que estão diretamente relacionadas com os seus serviços básicos, tanto administrativos quanto acadêmicos, tais como: e-mails; portais; sistemas de controle; workflows; fóruns; etc.

Diante dos resultados apresentados na Tabela 2, verifica-se que o estágio de implantação das práticas relacionadas à base tecnológica e funcional na organização pesquisada é ainda incipiente, o que demonstra uma baixa implantação de ferramentas de TI na IES X.

\subsection{Análise do Nível de Alcance nas Organizações das Práticas.}

$\mathrm{Na}$ análise do nível de alcance de cada uma das práticas ou ações relacionadas à base tecnológica e funcional, conforme Tabela 3, verifica-se que, para $47 \%$ dos coordenadores, as práticas e as ações relativas a Ferramentas de colaboração (portais, intranets e extranet) possuem poucas iniciativas isoladas dentro da organização ou alguns departamentos usam a prática. Entretanto, $35,3 \%$ dos coordenadores informaram que a prática é amplamente disseminada na IES X. Ainda que o nível de alcance da prática tenha sido baixo para $47 \%$ dos respondentes, identifica-se ampla disseminação na organização. A IES X conta com um portal que integra sistemas e gerencia o conhecimento e os dados da organização. Como a pesquisa evidenciou pouca aderência por parte dos respondentes, justifica-se a necessidade de maior comunicação interna e estímulo para disseminação da prática. 
Tabela 3: Nível de alcance de cada uma das práticas ou ações relacionadas à base tecnológica e funcional

\begin{tabular}{|c|c|c|c|c|c|}
\hline \multirow[b]{2}{*}{ AÇÃO OU PRÁTICA } & \multicolumn{5}{|c|}{ ALCANCE } \\
\hline & 1 & 2 & 3 & 4 & $N / R$ \\
\hline Ferramentas de colaboração como portais, intranets e extranets & 29,4 & 17,6 & 5,9 & 35,3 & 11,8 \\
\hline Sistemas de workflow & 17,6 & 23,5 & 17,6 & 29,4 & 11,8 \\
\hline Gestão de conteúdo & 64,7 & 11,8 & 17,6 & 0,0 & 5,9 \\
\hline Gestão Eletrônica de Documentos (GED) & 47,1 & 17,6 & 11,8 & 11,8 & 11,8 \\
\hline Data Warehouse (ferramenta da TI para apoio à GC) & 47,1 & 23,5 & 5,9 & 11,8 & 11,8 \\
\hline Data Mining (ferramenta da TI para apoio à GC) & 47,1 & 29,4 & 0,0 & 11,8 & 11,8 \\
\hline Costumer Relationship Management (CRM) & 17,6 & 35,3 & 5,9 & 17,6 & 23,5 \\
\hline Balanced Scorecard (BSC) & 35,3 & 5,9 & 11,8 & 5,9 & 41,2 \\
\hline Decision Suport System (DSS) & 29,4 & 11,8 & 17,6 & 0,0 & 41,2 \\
\hline Enterprise Resource Planning (ERP) & 23,5 & 17,6 & 11,8 & 5,9 & 41,2 \\
\hline Key Performance Indicator (KPI) & 29,4 & 11,8 & 11,8 & 5,9 & 41,2 \\
\hline Médias & 35,3 & 18,7 & 10,7 & 12,3 & 23,0 \\
\hline
\end{tabular}

Fonte: Elaborada pelos autores a partir da aplicação do questionário na IES X.

Com relação ao alcance da prática de Sistema de workflow, $47,1 \%$ dos coordenadores de curso informaram que ela é utilizada por muitos departamentos ou está amplamente disseminada na organização. Com isto, pode-se inferir que a prática se apresenta num estágio de alcance significativo. Isso se deve ao fato de a IES $X$ possuir um departamento específico para controle da qualidade dos processos operacionais que são utilizados pela organização para o desenvolvimento das atividades do Núcleo de Educação a Distância (NEAD). Desta forma, verifica-se que a IES X objetiva otimizar os processos por meio de sistema de workflow, formalizando os procedimentos e melhorando sua aplicação, diminuindo o tempo de atendimento e otimizando os trâmites necessários.

Salienta-se que nas práticas de gestão de conteúdo (76,5\%), GED (64,7\%), Data Warehouse $(70,6 \%)$, Data Mining $(76,5 \%)$ e CRM (52,9\%), existem poucas iniciativas isoladas dentro da organização ou alguns departamentos usam a prática.

Já no alcance da prática de gestão de conteúdo, o fato de esta prática ter sido identificada pela pesquisa como se apresentando apenas de maneira isolada para os coordenadores reflete um problema para a IES X. A prática é de interesse coletivo no que tange à disseminação do conhecimento e à busca de soluções para simplificação de processos organizacionais. Quando exercida de maneira efetiva, ela proporciona a disseminação das melhores práticas, tornando-se um canal de troca de informações pertinentes, possibilitando a melhora nos processos. Desta forma, ao utilizá-la de maneira isolada, a IES X não está fazendo o uso adequado desta prática, não potencializando o seu uso na instituição.

Quanto ao alcance da CRM, observa-se que ela se apresenta restrita a alguns departamentos da IES X, como na Diretoria de Relacionamento, que trata diretamente com o cliente. Por ser uma prática que contribui com o marketing de relacionamento com o cliente, torna-se evidente a importância de sua utilização no setor pedagógico. Conforme explicitado por Finger e Castro (2004), a prática personaliza o comportamento organizacional de acordo com as necessidades do cliente. Por meio da utilização da CRM no setor pedagógico, por exemplo, a IES X poderá personalizar 0 atendimento ao aluno, considerando as variáveis de comportamento levantadas pela prática CRM e, com isto, pode estreitar o vínculo entre as partes, algo que é relevante principalmente em se tratando da modalidade de EaD.

Em relação à prática ERP, esta se apresenta de maneira isolada e restrita a alguns departamentos, sendo pequeno o percentual de coordenadores que a utiliza. Mas apresentaram interesse em adotá-la.

Relacionado ao alcance da prática KPI e considerando que o nível de informações é volumoso nas organizações contemporâneas, a utilização de indicadores de desempenho auxilia no gerenciamento e na tomada de decisão. Na IES X, a prática KPI é utilizada para medir o índice de evasão dos cursos, o percentual de empréstimo de livros na biblioteca, entre outros. Ou seja, a prática é utilizada para o planejamento dos cursos. No entanto, a pesquisa evidenciou que entre os coordenadores de curso a utilização da prática ainda não é unânime. 
Os dados da Tabela 3 demonstram que as práticas que possuem o menor nível de alcance são justamente as práticas com menor grau de implementação. São estas: gestão de conteúdo, GED e Data Mining. Esta informação é importante, pois demonstra a necessidade de a IES X investir nestas práticas para melhorar 0 suporte à GC organizacional, e ampliar a automação da gestão da informação, dos aplicativos e das ferramentas de TI para captura, difusão e colaboração do conhecimento. Já como resultado positivo, verifica-se que a prática que atingiu o maior nível de alcance foram os sistemas de workflow. Esta prática é estratégica para a IES X por permitir o controle da qualidade da informação apoiado pela automação do fluxo ou trâmite de documentos e a instituição possui um grande volume de documentos, principalmente por operar na modalidade de EaD. Portanto, essa prática possui uma relação direta com os serviços básicos desempenhados pela IES X.

De forma geral, observa-se que o alcance aparece, principalmente, como restrito a alguns departamentos para todas as práticas. No entanto, há iniciativas isoladas presentes em todas as práticas para pelo menos $30 \%$ dos coordenadores de curso.

\section{CONSIDERAÇÕES FINAIS}

Como o foco do estudo foi analisar as práticas de GC relacionadas à base tecnológica e funcional da IES $X$, após análise dos resultados encontrados, é possível constatar que 0 estágio e 0 alcance das práticas relacionadas à base tecnológica funcional na IES X são ainda incipientes.

Segundo a análise do estágio de implantação das práticas relacionadas à base tecnológica e funcional apresentada na Tabela 2, o estágio atual da IES para os 17 coordenadores tem em média o seguinte perfil: para $27,3 \%$ não existem planos de implantação; $20,3 \%$ estão planejadas para o futuro; $15,5 \%$ estão em processo de implantação; $5,9 \%$ já estão implantadas; $12,3 \%$ estão implantadas e apresentando resultados relevantes. Observa-se que $18,7 \%$ dos coordenadores não responderam sobre estes quesitos. Portanto, percebe-se que 0 estágio de implantação das práticas relacionadas à base tecnológica e funcional da IES é ainda incipiente, pois apenas $18,2 \%$ das práticas estão implementadas e apresentando resultados. Isto significa que as práticas e as ferramentas de base tecnológica não estão dando o devido suporte para a captura, difusão e colaboração das informações e dos conhecimentos na instituição analisada.

Segundo análise do nível de alcance nas organizações das práticas relacionadas à base tecnológica $\mathrm{e}$ funcional apresentada na Tabela 3, o estágio atual da IES para os 17 coordenadores tem em média o seguinte perfil: $35,3 \%$ classificam como iniciativas isoladas; $18,7 \%$ acreditam que as práticas estão restritas a alguns departamentos; $10,7 \%$ acreditam que exista utilização das práticas; $12,3 \%$ acham que estão amplamente disseminadas na IES. Observa-se que $23 \%$ dos coordenadores não responderam sobre estes quesitos. Nota-se que o nível de alcance das práticas relacionadas à base tecnológica e funcional da IES é ainda incipiente, pois apenas $23 \%$ das práticas são utilizadas e disseminadas na IES, o que significa que as práticas de base tecnológica e funcional implantadas na instituição não estão sendo utilizadas com todo o seu potencial possível.

Analisando-se as práticas, observa-se que os maiores problemas foram verificados nas mesmas práticas tanto em relação ao nível de implementação quanto ao alcance, o que representa coerência com os resultados. São elas: gestão de conteúdo, GED e Data Mining. Isso demonstra uma baixa implantação de ferramentas de TI na IES X e necessidade da instituição investir nestas práticas para ampliar o suporte à GC organizacional e, com isto, tornar-se mais competitiva. Tal como foi discutido, para a IES X ser mais competitiva é fundamental que seus sistemas de informações que atuam nos processos, métodos e serviços da IES sejam eficientes e eficazes. As práticas que apresentaram os principais resultados (sistemas de workflow e o CRM) foram as que estão diretamente relacionadas com os serviços básicos da IES X, tanto administrativos quanto acadêmicos. Isto é relevante, pois demonstra que a IES analisada tem utilizado práticas e ferramentas de GC em base tecnológica que apenas contribuem para a execução da sua atividade fim, o que demonstra ampliação das possibilidades para a utilização dessas práticas.

Por fim, entende-se que existe necessidade de adoção de planos e estratégias voltadas exclusivamente para a implementação da GC pela instituição analisada, visando à consolidação destas práticas e ao seu uso em toda a sua potencialidade. Para trabalhos futuros, verifica-se a possibilidade de realizar uma análise comparativa entre o nível de implementação e alcance das práticas de GC relacionadas à base tecnológica e funcional da IES $X$ com a de outras IES. 


\section{REFERÊNCIAS}

AGUNE, R. O Governo no Século XXI. In: AGUNE, R.; GREGORIO, A.; NEVES; A.; DIAS, I. DE M.; CARLOS, J. A.; BOLLIGER, S. Dá pra fazer - Gestão do conhecimento e inovação em governo. São Paulo: Secretaria de Planejamento e Desenvolvimento Regional, 2014.

ALEE, V. The Knowledge Evolution: Expanding Organizational Intelligence. Newton: Butterworth-Heinemann, 1997.

ANGELONI, M.T. Organizações do conhecimento: infraestrutura, pessoas e tecnologias. São Paulo: Saraiva, 2002.

BATISTA, F. F. Modelo de gestão do conhecimento para a administração pública brasileira: como implementar a gestão do conhecimento para produzir resultados em benefício do cidadão. Brasília: Ipea, 2012.

BATISTA, F.F. $O$ desafio da gestão do conhecimento nas áreas de administração e planejamento das instituições federais de ensino superior. Texto para Discussão, $n^{\circ}$ 1181. Brasília: Ipea, 2006.

BATISTA, F.F.; QUANDT, C.O.; PACHECO, F. F.; TERRA, J. C. C. Gestão do conhecimento na administração pública. Texto para Discussão, n’ 1095. Brasília: Ipea, 2005.

BATISTA, F. F; RECH, A R., GOMES, C. de A.; SANTOS, D. L. N. dos, ANDRADE, E. C. S. de; MALLMANN, M. L; FERREIRA, R. M. P. M.; COSTA, V. da S. Casos Reais de implantação do modelo de gestão do conhecimento para a administração pública brasileira. Texto para Discussão, n 1941. Brasília: Ipea, 2014.

BATISTA, F. F.; XAVIER, A. C. da R.; MENDES, L. C.; ROSENBERG, G. GC em organizações públicas de saúde. Texto para Discussão, n¹316. Brasília: Ipea, 2007

BATISTA, F.F. Modelo de Gestão do Conhecimento para a Administração Pública Brasileira: como implementar a Gestão do Conhecimento para produzir resultados em benefício do cidadão. Rio de Janeiro: Ipea, 2012.

BENTO, J. C.; MASSUDA, E. M; URPIA, A. G. B. da C.; BORTOLOZZI, F. Práticas da gestão do conhecimento em recursos humanos em instituição de ensino superior à distância. Espacios, v.37, n.29, p.21-30, 2016.

BOIKO, B. Content Management Bible. New York: John Wiley \& Sons, Inc., 2001.

BOLSON, S. B. Gestão do Conhecimento: estudo em uma IES Tecnológica. Dissertação (Mestrado) Universidade Potiguar, Natal, 2012.

BRITO, L. M. P.; GALVÃO, A. P.; OLIVEIRA, P. W. S. Gestão do Conhecimento em Empresa Internacional de Energia. Informação \& Sociedade: Estudos, v.23, n.1, p.93-103, 2013.

CHAPARRO, F. Apropiacion social del conocimiento em el processo de construccion de sociedade. In: Simpósio de Gestão da Inovação Tecnológica. Anais... São Paulo: ANPAD, 1998.

BUCKHOUT, S.; FREY, E.; NEMEC JR., J. Por um ERP eficaz. HSM Management. p.30-36,1999.

$\mathrm{CHOO}, \mathrm{C}$. W. A organização do conhecimento: como as organizações usam a informação para criar significado, construir conhecimento e tomar decisões. São Paulo: Ed. SENAC, 2003.

COUTINHO, L. G.; FERRAZ, J. C. Estudo de Competitividade da Indústria Brasileira, $3^{\mathrm{a}}$ edição, Campinas: Papirus, 1995.

DAVENPORT, T.H.; PRUSAK, L. Conhecimento Empresarial: como as organizações gerenciam o seu capital intelectual. Rio de Janeiro: Campus, 1998.

DAVENPORT, T. H.; DE LONG, D. W.; BEERS, M. C. Successful Knowledge Management Projects. Sloan Management Review, p. 43-57, 1998.

DRUZDZEL, M. J.; FLYNN, R. R. Decision Support Systems. Encyclopedia of Library and Ciência da Informação. 2. ed. Nova lorque: Marcel Dekker, Inc, 2002.

ELMASRI, R.; NAVATHE, S. B. Sistemas de banco de dados: fundamentos e aplicações. Rio de Janeiro: LTC, 2002. 
FACHIN, G.R.B.; STUMM, J.; COMARELLA, R.L.; FIALHO, F. A. P.; SANTOS, N. dos. Gestão do conhecimento e a visão cognitiva dos repositórios institucionais. Perspectivas em Ciência da Informação, v. 14, p. 220-236, 2009.

FIGUEIRA, M.; GONÇALVEZ, E. J. V.; SUGANO, J. Y.; ZAMBALDE, A. L. Inovação organizacional e inovação de modelo de negócios: um estudo comparativo. Revista Espacios, v. 32, n. 3, p. 10-12, 2011.

FINGER, Andrew B.; CASTRO, Gardênia de. Mudança na gestão das bibliotecas universitárias públicas através da implementação do Customer Relationship Management (CRM). In: Seminário Nacional de Bibliotecas Universitárias. Anais... Natal: UFRN, 2004.

FREIRE, P. S.; UENO, A. T.; DIAS, M. A. H.; SANTOS, N. dos. Ferramentas de avaliação de Gestão do Conhecimento: Um estudo bibliométrico. International Journal of Knowledge Engineering \& Management, v.2, n.3, p. 15-38, 2013.

FRESNEDA, P. S. V.; GONÇALVES, S. M. G.; PAPA, M.; FONSECA, A. F. Diagnóstico da Gestão do Conhecimento nas Organizações Públicas Utilizando o Método OKA. In: II CONSAD de Gestão Pública. Anais... Brasilia, 2009.

KAPLAN, R.S.; NORTON, D. P. A estratégia em ação: Balanced Scorecard. Rio de Janeiro: Campus, 1997. KIMBALL, R. Data wirehouse toolkit. São Paulo. Makron Books, 1998.

KING, D. Numerical machine learning. Technical Report CS 4803B, Georgia: Tech College of Computing, 2003.

KOCH, W. W. Gerenciamento eletrônico de documentos: conceitos, tecnologias e considerações gerais. São Paulo: Cenadem, 1997.

MENDES, R. B. Análise do nível de maturidade da gestão do conhecimento na criminalística federal. Dissertação (Mestrado), Fundação Getúlio Vargas, Rio de Janeiro, 2013.

NADER, B.; TOMI, G.; PASSOS, A. O. Indicadores-chave de desempenho e a gestão integrada da mineração. Revista Escola de Minas, v.65, n.4, 2012.

NATALE, C. H. C.; NEVES, J. T. de R. Metodologia para identificação de modelos de maturidade em gestão do conhecimento para a aplicação empírica. In: XXXVIII Encontro da ANPAD. Anais... Rio de Janeiro, p. 1-12, 2014.

NONAKA I.; TAKEUCHI, H. Criação de conhecimento na empresa. Rio de Janeiro: Elsevier, 1997.

NONAKA I.; TAKEUCHI, H. Gestão do Conhecimento. Porto Alegre: Bookman, 2008.

OLIVEIRA, E. A. de A.; CARVALHO, A. R. de S. Ferramentas de disseminação do conhecimento em uma instituição pública de C,T\&I de defesa da nação. LAJBM, v. 6, n. 1, p. 178-196, edição especial 1/2015, Taubaté, SP, Brasil.

PARREIRAS, F.S. Geração de Sistemas de Gestão de Conteúdo com Softwares Livres. Perspectivas em Ciência da Informação, v.10, n,2, p.240-252, 2005.

PORTER, M. E. Competição: estratégias competitivas essenciais. 7. ed., Rio de Janeiro: Campus, p. 27 - 45, 1999.

POSSOLLI, G.E. Gestão da Inovação e do Conhecimento. Curitiba: InterSaberes, 2012.

RODRIGUES, M. M.; GRAEMI, A. R. Conhecimento tácito ou explícito? a dimensão epistemológica do conhecimento organizacional na pesquisa brasileira sobre gestão do conhecimento. Perspectivas em Gestão \& Conhecimento, v. 3, n. 2, p. 131-144, jul./dez. 2013.

SCHREIBER, D. O Estudo da Influência Simbólica do Líder no Processo de Gestão do Conhecimento. Revista Gestão \& Tecnologia, v.15, n.1, p.200-229, 2015.

SCHUMPETER, J. A. Teoria do Desenvolvimento Econômico: uma investigação sobre lucros, capital, crédito, Juro e o ciclo econômico. São Paulo: Nova Cultural, 1997. 
STEFANO, N. M.; CASAROTTO FILHO, N.; FREITA, M. do C. D.; MARTINEZ, M. A. T. Gestão de ativos intangíveis: implicações e relações da gestão do conhecimento e capital intelectual. Perspectivas em Gestão \& Conhecimento, v. 4, n. 1, p. 22-37, jan./jun, 2014.

SULLIVAN, K. O. Leveraging knowledge management technologies to manage intellectual capital. In: STANKOSKY, M. Creating the discipline of knowledge management the latest in university research. Oxford: Elsevier Butterworth-Heinemann, 2005.

TEIXEIRA FILHO, J. Gerenciando Conhecimento: como a empresa pode usar a memória organizacional e a inteligência competitiva no desenvolvimento de negócios. Rio de Janeiro: Ed. SENAC, 2000.

YOUNG, R. Knowledge Management: tools and techniques manual. Asian Productivity Organization. Tokio, 2010.

URPIA, Arthur Gualberto B. da C.; MASSUDA, Ely Mitie; BORTOLOZZI, Flavio; FORNO, Leticia Fleig Dal.; MACUCH, Regiane da Silva. A gestão do conhecimento nas escolas da Amusep. In.: MENEGASSI, Cláudia Herrero Martins; BORTOLOZZI, Flávio; JUNIOR, Nelson Tenório; SARTORI, Rejane. (Orgs.). Gestão do conhecimento nas organizações: Inovação, Educação, tecnologias e Gestão. São Paulo: Gregory, 2016. 